\title{
Ein Teuthiden-Rest aus dem Obernor (Kössener-Schichten) der Lahnewies-Neidernachmulde bei Garmisch-Partenkirchen (Bayern)
}

\author{
JOACHIM ReITNer, Tübingen *
}

Mit 6 Abbildungen und 1 Tabelle im Text

\begin{abstract}
A bstract: A description of a new Teuthida in the Upper Triassic (Kössen-Beds) from the Lahnewies-Neidernachsyncline (Garmisch-Partenkirchen, Bavaria) is given. Loligosepia neidernachensis $\mathrm{n}$. sp. is probably a forerunner of the early liassic forms of Teuthida.

$\mathrm{Z}$ us ammenfassung: Beschrieben wird eine neue Teuthiden-Art aus der Obertrias (Kössener-Schichten) der Lahnewies-Neidernachmulde (Garmisch-Partenkirchen, Bayern). Loligorepia neidernachensis n. sp. kann als Ausgangsform der liassischen Teuthiden angesehen werden.
\end{abstract}

\section{Einleitung}

Die Überlieferung von Endocochleaten, mit Ausnahme der Belemnoideen, stellt eine große Seltenheit dar. Die zarten Schalenreste werden, soweit sie aus organischer Substanz bestehen, postmortal durch Verwesung abgebaut und gehen daher nicht in den fossilen Bericht ein. Die nur schwach mineralisierten Schulpe werden wegen ihrer Feinheit bei der Diagenese meist aufgelöst.

Bisher sind Teuthiden-Reste seit dem Lias bekannt. Bei systematischen Fossilaufsammlungen in den Kössener-Schichten der Lahnewies-Neidernachmulde bei Garmisch-Partenkirchen wurde der Abdruck des hier beschriebenen Teuthiden-Restes gefunden, bei dem es sich um den bisher ältesten Fund dieser Dibranchiaten-Gruppe handelt.

An dieser Stelle möchte ich Herrn Fotograf Wetzel (Tübingen) für die Anfertigung der Fotos danken. Herrn Dr. DieTl (Ludwigsburg), Herrn Dr. URLICHS (Ludwigsburg) und Herrn Präparator LörCHER (Dotternhausen, Portland Zementwerk) danke ich für Einblicke und Überlassung von Vergleichsmaterial. Mein besonderer Dank gilt Herrn Prof. WIEDManN (Tübingen) und Herrn Dr. IMmel (Tübingen) für wertvolle Anregungen und Durchsicht des Manuskripts.

*Anschrift des Verfassers: Cand. geol. JoАсhim Reitner, Geol.-Paläont. Institut der Universität, Sigwartstraße 10, 7400 Tübingen 1 . 


\section{Systematik}

Unterklasse: Coleoidea BATHER 1888

Ordnung: Teuthida NaEf 1916

Unterordnung: Loligosepiina JELETZKY 1965

Familie: Loligosepiidae van Regteren Altena 1949

Loligosepia neidernachensis $\mathrm{n}$. $\mathrm{sp}$.

Abb. 1, Abb. 3 a, b

De rivatio nomin is: Nach dem Fundort am Flüßchen Neidernach bei Griesen (Kreis Garmisch-Partenkirchen, Bayern).

Holoty pus : GPIT Nr.1529/1 a, b.

Locus typicus: Wegkreuzung am nördlichen Neidernach-Ufer, ca. $2 \mathrm{~km}$ W der Zoll. station Griesen.

$\mathrm{Stratum}$ typicum : Eine $6 \mathrm{~cm}$ dicke Bank aus einem mittel- bis dunkelgrauen, feinlaminierten, leicht bituminösen Mergel mit Durchwühlungsspuren. Die Bank befindet sich direkt unterhalb des Lagers von Eopsiloceras planorboides (GüMBEL) der Lahnewies-Neidernachmulde. Die Bank enthält Choristoceras rhaeticum (GüMBEL) und Choristoceras ammonitiforme (GüMBEL) und ist mit dem überlagernden Eopsiloceras-Niveau in das Obersevat (Nor) zu stellen. Charakteristisch für die Bank ist das massenhafte Auftreten kleinwüchsiger Choristoceraten in aus-

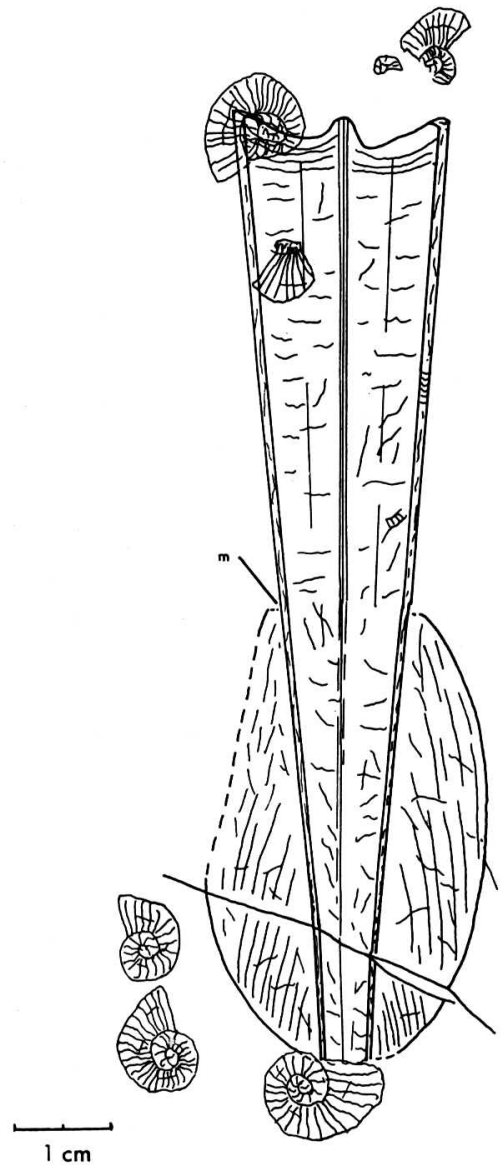

Abb. 1. Loligosepia neidernachensis n. sp., Ansicht von der Ventralseite. Spitzes Ende des Gladius ist unvollständig. Die mediale Asymptote ist gut zu erkennen $(\mathrm{m}) ; \times 1$.

Fig. 1. Loligosepia neidernachensis n. sp., ventral view. The peak end of the gladius is not preserved. The medial asymptote is easily recognizable (m); $\times 1$. 
gezeichneter Aragoniterhaltung. Ca. 40 Prozent der Exemplare besitzen noch die Anaptychen in situ. Zusätzlich finden sich zahlreiche kleinwüchsige Pectiniden. Alle Fossilien sind ausnahmslos verdrückt.

Materia 1: 1 Exemplar.

Inventarnummern Geol.-Paläont. Inst. Tübingen (GPIT):

Geopeltis simplex - 1. Nr. 1529/5;2. Nr. 1529/6; 3. Nr. 1529/3

Loligosepia aalensis - 1. Nr. 1529/7; 2. Nr. 1529/8; 3. Nr. 1529/9; 4. Nr. 1529/10; 5 . Nr. 1529/12; 7. Nr. 1529/14

Inventarnummmern Staatl. Mus. Naturk. Stuttgart (SMNS):

Geopeltis simplex - 1. Nr. 4023; 2. Nr. 21808; 3. Nr. 23687; 4. Nr. 23686

Loligosepia aalensis - 1. Nr. 21807; 2. 23684; 3. Nr. 23687

Di a g n o s e : Ein Teuthide mit relativ schlankem Gladius, dessen Konusfahnen die hintere Hälfte des Gladius begleiten. Die Mittelleiste ist als schwacher Kiel ausgebildet, der jederseits von einem feinen parallelen Streifen begrenzt wird.

B es chreibung: Auf einer ca. $18 \times 23 \mathrm{~cm}$ großen Mergelplatte findet sich der Abdruck eines schwach kalzifizierten Dibranchiaten-Schulpes. Ebenso liegt auch der Gegenabdruck vor. Der Schulp selbst ist bis auf sein spitzes Ende vollständig erhalten. Er besitzt eine Länge von $91 \mathrm{~mm}$; die ursprüngliche Länge läßt sich rekonstruieren und hat vermutlich $112 \mathrm{~mm}$ betragen. Die maximale Breite des Gladius beträgt $23 \mathrm{~mm}$. Der Gladius hat einen Apikalwinkel von ca. $10^{\circ}$. Die Randstreifen zeigen eine BogenstreifenStruktur. Sie sind schwach ausgebildet, ihre Breite beträgt ca. 1,5 mm. Die Mittelleiste des Gladius ist als schwacher Kiel ausgebildet und wird beiderseits von einem parallelen, sehr feinen Streifen begrenzt. Der Kiel hebt sich am breiten Ende des Gladius zeltförmig ab, was an Paraplesioteuthis sagittata (Münster) (Abb. 6) erinnert. Die Mittelplatten zeigen zu beiden Seiten der Mittelleiste konkave Anwachslinien und feine Leisten, die parallel zum Kiel verlaufen. Die Konusfahnen besitzen eine Länge von $52 \mathrm{~mm}$. Ihr Anwachsbereich an den Gladius ist nur schlecht erhalten. Ein Hyperbolarfeld läßt sich wegen der schlechten Erhaltung nur vermuten. Die mediale Asymptote ist gut zu erkennen, die laterale hingegen nur undeutlich. Die Konusfahnen zeigen eine Anwachsstruk-

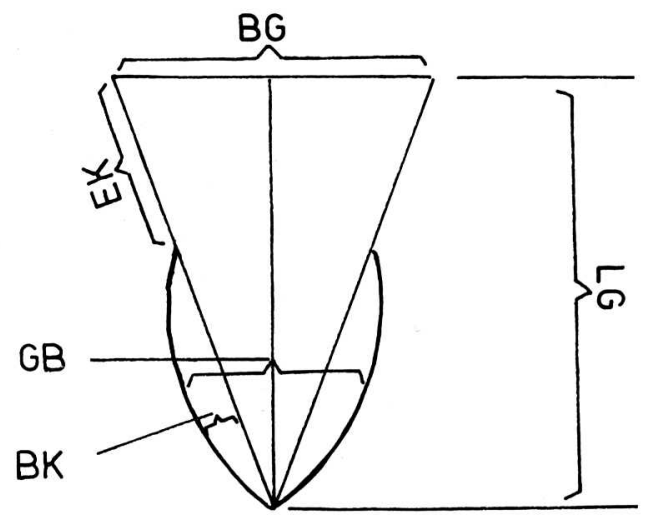

Abb. 2. Meßwerteschema. Um vergleichbare Größen zu bekommen, wurden folgende Parameter gewählt: Länge des Gladius: LG; Breite des Gladius: BG; Breite der Konusfahnen: BK; Gesamtbreite der Konusfahnen und des Gladius: GB; Einsetzen der Konusfahnen: EK. - Verhältnisse: LG : EK; LG : BG; LG : GB; GB : BK.

Fig. 2. Measuring scheme. The following parameters were selected in order to get comparative values: Length of the gladius: LG; Breadth of the gladius: BG; Breadth of the wings: BK; Breadth of the wings and of the gladius: GB; Indentation of the wings: EK. - Proportions: LG : EK; LG : BG; LG : GB; GB : BK. 
tur, die zur Ventralseite des Schulps hinweisen. Eine Hälfte der Konusfahnen weist eine maximale Breite von $12 \mathrm{~mm}$ auf. Dagegen beträgt die Gesamtbreite der Konusfahnen und des Gladius $31 \mathrm{~mm}$.

Differentialdiagnose: Von den liassischen Arten Loligosepia bucklandi (VOLTZ) und Loligosepia aalensis (ZIETEN) unterscheidet sich Loligosepia neidernachensis n. sp. durch den einfachen Kiel, die geringe Größe der Konusfahnen und vor allem durch die wenig differenzierten Mittelplatten.

Tab. 1. Vergleichswerte der frühen Teuthiden aus dem Toarc von Schwaben und von Loligosepia neidernachensis n. sp. von Garmisch-Partenkirchen.

Tab. 1. Comparative values of the early teuthids in the Toarcian of Swabian and Loligosepia neidernachensis n. sp. of Garmisch-Partenkirchen.

\begin{tabular}{|c|c|c|c|c|c|}
\hline & $\begin{array}{c}\text { Loligosepia } \\
\text { neidernachensis }\end{array}$ & $\begin{array}{r}\text { Geopeltis } \\
\text { Slg. SMNS }\end{array}$ & $\begin{array}{l}\text { simplex } \\
\text { Slg. GPIT }\end{array}$ & $\begin{array}{l}\text { Loligosepia } \\
\text { Slg. SMNS }\end{array}$ & $\begin{array}{l}\text { aalensis } \\
\text { Slg. GPIT }\end{array}$ \\
\hline LG : EK & 2,46 & $\begin{array}{l}\text { 1. } 3,52 \\
\text { 2. } 3,86 \\
\text { 3. } 4,00 \\
\text { 4. } 3,63\end{array}$ & $\begin{array}{l}\text { 1. } 2,95 \\
\text { 2. } 3,52 \\
\text { 3. } 3,72\end{array}$ & $\begin{array}{l}\text { 1. } 8,53 \\
\text { 2. } 5,66 \\
\text { 3. } 6,66\end{array}$ & $\begin{array}{l}\text { 1. } 5,40 \\
\text { 2. } 6,87 \\
\text { 3. } 5,28 \\
\text { 4. } 5,92 \\
\text { 5. } 4,00 \text { ? } \\
\text { 6. } 4,33 \\
\text { 7. } 8,33\end{array}$ \\
\hline LG : BG & 5,04 & $\begin{array}{l}\text { 1. } 1,90 \\
\text { 2. } 2,50 \\
\text { 3. } 2,58 \\
\text { 4. } 2,52\end{array}$ & $\begin{array}{l}\text { 1. } 2,44 \\
\text { 2. } 2,50 \\
\text { 3. } 2,36\end{array}$ & $\begin{array}{l}\text { 1. } 3,96 \\
\text { 2. } 2,93 \\
\text { 3. } 3,33\end{array}$ & $\begin{array}{ll}\text { 1. } 2,36 \\
\text { 2. } 2,89 \\
\text { 3. } 2,40 \\
\text { 4. } 2,96 \\
\text { 5. } 4,00 \text { ? } \\
\text { 6. } 3,56 \\
\text { 7. } 2,55\end{array}$ \\
\hline LG : GB & 3,58 & $\begin{array}{l}\text { 1. } 1,78 \\
\text { 2. } 2,11 \\
\text { 3. } 1,92 \\
\text { 4. } 2,68\end{array}$ & $\begin{array}{l}\text { 1. } 2,21 \\
\text { 2. } 2,32 \\
\text { 3. } 1,96\end{array}$ & $\begin{array}{l}\text { 1. } 5,12 \\
\text { 2. } 3,03 \\
\text { 3. } 3,22\end{array}$ & $\begin{array}{ll}\text { 1. } & 3,89 \\
\text { 2. } & 4,15 \\
\text { 3. } 2,89 \\
\text { 4. } 3,72 \\
\text { 5. } 2,86 ? \\
\text { 6. } 4,56 \\
\text { 7. } 2,55\end{array}$ \\
\hline $\mathrm{GB}: \mathrm{BK}$ & 2,58 & $\begin{array}{l}\text { 1. } 3,20 \\
\text { 2. } 3,00 \\
\text { 3. } 3,60 \\
\text { 4. } 3,08\end{array}$ & $\begin{array}{l}\text { 1. } 3,27 \\
\text { 2. } 3,30 \\
\text { 3. } 3,29\end{array}$ & $\begin{array}{l}\text { 1. } 3,42 \\
\text { 2. } 2,80 \\
\text { 3. } 3,10\end{array}$ & $\begin{array}{ll}\text { 1. } 3,11 \\
\text { 2. } 2,94 \\
\text { 3. } 2,90 \\
\text { 4. } 3,30 \\
\text { 5. } 3,50 \text { ? } \\
\text { 6. } 3,00 \\
\text { 7. } 2,57\end{array}$ \\
\hline
\end{tabular}

\section{Phylogenetische Beziehungen}

Bei dem vorliegenden Stück handelt es sich um den bisher ältesten Fund eines Teuthiden.

Zu der phylogenetisch älteren Gruppe der Phragmoteuthida aus der unteren Obertrias (Karn) zeigen sich keine unmittelbaren Beziehungen, obwohl sich die Merkmale des Proostrakums durchaus mit denen der frühen liassischen Teuthiden homologisieren lassen 
(Jeletzky 1966: 32 ff.). Das Fehlen eines Phragmokons und die relativ schlanke Form des Gladius sind sichere Merkmale echter Teuthiden.

Die ältesten bis jetzt bekannten Teuthiden kennt man aus dem Sinemur von England (Jeletzky 1966; Buckland 1840: Taf. 28, Abb. 6-7; Taf. 29, Abb. 1-3; Voltz 1840: Taf. 30). Bei den Stücken handelt es sich meistens um die Art Loligosepia bucklandi (VolTz). Die reichsten Funde früher Teuthiden kennt man aus dem Toarc von Schwaben. Hier treten bereits mehrere Unterordnungen auf. Am besten läßt sich die neue Art mit den ältesten Arten der liassischen Gattung Loligosepia vergleichen. Es zeigen sich Ähnlichkeiten in der Ausbildung der Mittelleiste und in der schlanken Form des Gladius (kleiner Apikalwinkel). Die Größe der Konusfahnen könnte für Zugehörigkeit zu der Gattung Geopeltis sprechen. Doch ist dieses Merkmal von nur geringer Bedeutung, da es innerhalb einer Art sehr variieren kann.

Von den liassischen Loligosepiida weicht die neu beschriebene Art nur wenig ab. Es ist daher denkbar, daß sie an der Wurzel der Radiation der liassischen Teuthiden steht.
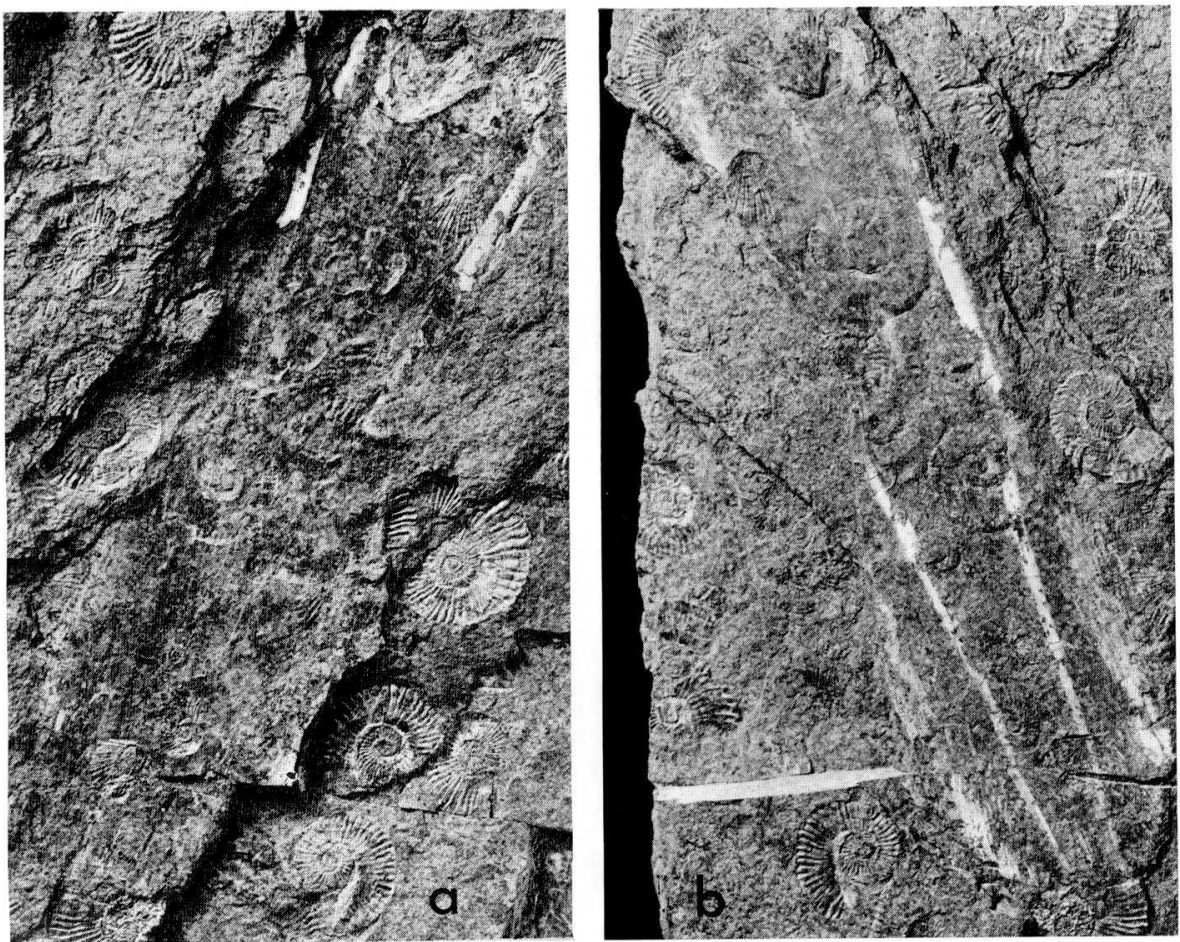

Abb. 3 a-b. Loligosepia neidernachensis n. sp., Holotyp, Kössener Schichten (Obernor) von Garmisch-Partenkirchen (GPIT 1529/1 a, b). Abdruck und Gegenabdruck mit Choristoceras sp. und kleinwüchsigen Pectiniden; $\times 1$.

Fig. 3 a-b. Loligosepia neidernachensis n. sp. holotypus, Kössen Beds (Upper Norian) from Garmisch-Partenkirchen (GPIT $1529 / 1 \mathrm{a}, \mathrm{b}$ ). The specimen (fossil and cast) with Choristoceras sp. and small pectinids; $\backslash 1$. 

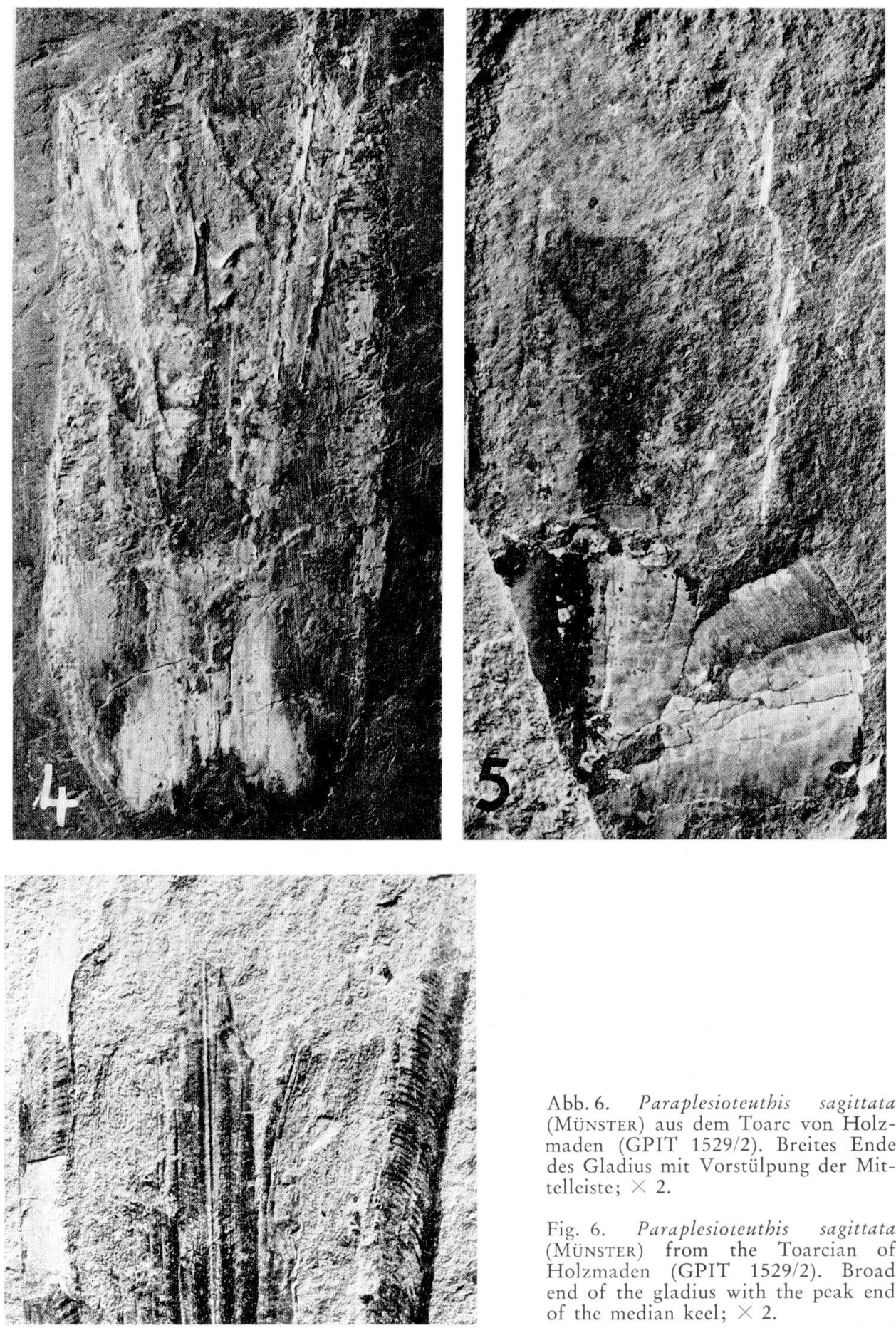

Abb. 6. Paraplesioteuthis sagittata (Münster) aus dem Toarc von Holzmaden (GPIT 1529/2). Breites Ende des Gladius mit Vorstülpung der Mittelleiste; $\times 2$.

Fig. 6. Paraplesiotenthis sagittata (Münster) from the Toarcian of Holzmaden (GPIT 1529/2). Broad end of the gladius with the peak end of the median keel; $\times 2$. 


\section{4. Ökologie}

Die Erhaltungsbedingungen im Bereich des Eopsiloceras-Lagers der Kössener-Schichten waren optimal. Dokumentiert wird dies vor allem durch die ausgezeichnete Erhaltung der Ammonoideen, die aus derselben Bank stammen. Die Ursache liegt vermutlich in den schwach euxinischen Verhältnissen der Sedimentbecken der Hinterriff-Bereiche der Obertrias. Dafür sprechen der schwache Bitumengehalt der Mergel, gelegentliche Pyritkonkretionen und der in der Lahnewiesmulde in etwa gleichem stratigraphischem Niveau gefundene "Ölschiefer «.

Trotz jahrelanger Fossilaufsammlungen wurde nur dieses eine Stück gefunden. Eine Erklärung dafür wäre, daß die Teuthiden zum Pelagial gehörten und so nur selten in die Lagunen im Hinterriff-Bereich verdriftet wurden.

\section{Literatur}

BronN, H. G. (1859): Nachtrag über die Trias-Fauna von Raibl. - N. Jb. Geol. Paläont., 1859: 39-45, Taf. 1; Stuttgart.

BúcKLAND, W. (1836): Geology and Mineralogy considered with reference to natural Theology. -2 Bde., 1 (Text), 618 S., 2 (Tafeln), 128 S., 69 Taf.; London (Pickering Press).

Diener, C. (1915): Cephalopoda triadica. - Foss. Cat. I., Animalia, 8: 369 S.; Berlin (W. Junk). HöLdER, H. (1973): Ein belemnitisches Proostrakum aus dem Unteren Lias. - In: HöldDER, H.: Miscellanea cephalopodica. - Münster. Forsch. Geol. Paläont., 29: 63-67, Abb. 10, Taf. 2, Fig. 7; Taf. 3, Fig. 8; Münster (Westf.).

JELETZKy, J.A. (1966): Comparative morphology, phylogeny and classification of fossil coleoidea. - Univ. Kansas Paleont. Contrib., Mollusca, Art. 7: 162 S., 15 Abb., 25 Taf.; Lawrence, Kansas.

Mojsisovics, E. v. (1882): Die Cephalopoden der mediterranen Triasprovinz. - Abh. k.k. geol. Reichsanst., 10: 322 S., 94 Taf.; Wien.

- (1902): Das Gebirge um Hallstatt. Die Cephalopoden der HallstätterKalke. Bd.1, Suppl. - Abh. k. k. geol. Reichsanst., 4 (1. Hälfte): 175-256, Taf. 1-23; Wien.

MüLlER, A.H. (1965): Lehrbuch der Paläozoologie. Bd. II: Invertebraten, Teil 2, Mollusca 2 Arthropoda 1. -502 S., 668 Abb.; Jena (G. Fischer).

- (1967): Ein (?) Dibranchiatenrest (Triadoteuthis parvula n. g. n. sp., ? Cephalopoda) aus dem Oberen Muschelkalk (Ceratitenschichten) von Ballenstedt. - Geologie, 1967: 74-79, 3 Abb., 4 Taf.; Berlin.

NafF, A. (1922): Die fossilen Tintenfische. - 322 S., 101 Abb.; Jena (G. Fischer).

Abb. 4. Loligosepia aalensis (ZIETEN) von Holzmaden (GPIT 1529/4). Die Konusfahnen sind vollständig erhalten. Das breite Ende des Gladius ist nicht erhalten. Das Hyperbolarfeld ist gut erkennbar; $\times 1$.

Fig. 4. Loligosepia aalensis (ZIETEN) of Holzmaden (GPIT 1529/4). The wings are completely preserved. The broad end of the gladius is not preserved. The hyperbolar field is easily recognizable; $\times 1$.

Abb. 5. Pbragmoteuthis bisinuata (BRONN) aus den Raibler Schichten der Schartenklamm bei Raibl (Ósterreich) (SMNS 23689). Die linke Konusfahne ist nicht erhalten. Der dunkle Umriß auf dem Proostrakum ist wahrscheinlich der Abdruck des Tintenbeutels. Die Mittelleiste ist schwach erkennbar. Ein Hyperbolarfeld ist nicht erhalten. Der Phragmokon ist zerbrochen; $\times 2$.

Fig. 5. Pbragmotentbis bisinuata (BRONN) in the Raibl Beds from the Schartenklamm near Raibl (Austria) (SMNS 23689). The left wing is not preserved. The dark outline on the proostracum is probably the remains of an inkbag. The median keel is indestinate. A hyperbolar field is not preserved in this specimen, while the phragmocone is broken; $\times 2$. 
Quenstedt, F. A. (1839): Loligo Bollensis ist kein Belemniten-Organ. - N. Jb. Mineral. Geol. Paläont., 1839: 156-167, 1 Abb.; Stuttgart.

- (1849): Petrefactenkunde Deutschlands. Bd. 1. Die Cephalopoden. - 581 S., Atlas 36 Taf.; Tübingen (Fues).

- (1885): Handbuch der Petrefactenkunde. 3. Aufl. - 1239 S., Atlas 100 Taf.; Tübingen (Laupp).

Regteren Altena, C. O. van (1947): Systematic catologue of the paleontological collection. Sixth Suppl., Teuthoidea. - Mus. Teyler Arch., (3), 10: 53-62, 1 Abb., 1 Taf.; Haarlem.

Rieber, H. (1970): Phragmoteuthis ? ticinensis n. sp., ein Coleoidea-Rest aus der Grenzbitumenzone (Mittlere Trias) des Monte San Giorgio (Kt. Tessin, Schweiz). - Paläont. Z., 44: 32-40, 3 Abb., Taf. 3-4; Stuttgart.

Suess, E. (1865): Úber die Cephalopoden-Sippe Acanthoteuthis R. WAGN. - Sitz.-Ber. Akad. Wiss., math. nat. Kl., 51, 1 . Abt., Jg. 1865: 225-244, Taf. 1-4; Wien.

Voltz, P. L. (1830): Observations sur les belemnites. - Mém. Soc. Mus. hist. Nat., 1: 70 S., 8 Taf.; Paris.

WiedmanN, J: (1972): Ammoniten-Nuklei aus Schlämmproben der nordalpinen Obertrias — ihre stammesgeschichtliche und stratigraphische Bedeutung. - Mitt. Ges. Geol. Bergbaustud., 21 : 561-613, 21 Abb., 1 Tab., 6 Taf.; Innsbruck.

Zieten, A. (1830): Die Versteinerungen Württembergs. Expeditum des Werkes »Unsere Zeit«. — 102 S., 22 Taf.; Stuttgart.

Eingang des Manuskriptes bei der Schriftleitung am 22. 3. 1978. 